Review began 10/17/2021 Review ended 11/25/2021 Published 11/28/2021

() Copyright 2021

Shah et al. This is an open access article distributed under the terms of the Creative Commons Attribution License CC-BY 4.0., which permits unrestricted use, distribution, and reproduction in any medium, provided the original author and source are credited.

\section{A Retrospective, Observational Study to Assess the Efficacy, Safety, and Tolerability of Dietary Fiber Supplemental Combination in Overweight or Obese Patients}

\author{
Ami Shah ${ }^{1}$, Sabiha Siddiqui ${ }^{2}$, Salome Benjamin ${ }^{3}$, Mansi Patil ${ }^{4}$, Himanshu P. Tayade ${ }^{5}$ \\ 1. Dietetics, DietCastle Clinic, Mumbai, IND 2. Department of Nutrition, Parsee General Hospital, Mumbai, IND 3. \\ Department of Nutrition, Jupiter Hospital, Thane, IND 4. Department of Nutrition, Marian Cardiac Center, Pune, IND 5. \\ Pharmacology, Mediscience Healthcare Communications Private Limited, Navi Mumbai, IND
}

Corresponding author: Ami Shah, ami.dietetics@gmail.com

\section{Abstract \\ Study Objective}

To assess the efficacy, safety, and tolerability of dietary fiber supplementation combination (DFSC) in decreasing the bodyweight (BW) and body mass index (BMI) of obese or overweight patients.

\section{Methods}

This was a retrospective, observational, multicentric, prescription event monitoring study. Forty-two overweight to obese individuals consumed DFSC, a combination of inulin, partially hydrolyzed guar gum, and resistant maltodextrin along with dietary and physical activity interventions. The cases had the following diet intake: $45 \%-55 \%$ carbohydrate, $15 \%-20 \%$ protein, and 20\%-25\% fat, with $15 \mathrm{~g}$ visible fat/day and 18-24 g DFSC daily for 12 consecutive weeks with vigorous diet monitoring fortnightly.

\section{Results}

The mean age of the patients was 40.74 years (standard deviation (SD): 12.16). The mean bodyweight and BMI of the patients were $80.63 \mathrm{~kg}$ (SD: 14.34) and $32.24 \mathrm{~kg} / \mathrm{m}^{2}$ (SD: 13.98), respectively, at the baseline. At the end of weeks 4,8 , and 12 , diet therapy and DFSC showed statistically significant reductions in the mean bodyweight and BMI as follows: $3.03 \mathrm{~kg}$ (SD: 01.24) and $1.18 \mathrm{~kg} / \mathrm{m}^{2}(\mathrm{SD}: 00.52)(p=0.001), 5.70 \mathrm{~kg}$ (SD: 02.21 ) and $2.31 \mathrm{~kg} / \mathrm{m}^{2}$ (SD: 01.08) $(p=0.001)$, and $7.82 \mathrm{~kg}$ (SD: 03.06) and $3.27 \mathrm{~kg} / \mathrm{m}^{2}$ (SD: 01.86) $(p=0.001)$, respectively. Healthcare professionals rated diet therapy and DFSC as good to excellent for their efficacy and safety in $97.6 \%$ of the cases, and adverse event was not reported in any case with DFSC.

\section{Conclusion}

Dietary fiber supplemental combination with proper diet therapy/modification was found to be safe and effective in causing significant weight reduction in obese or overweight patients. However, a large multicentric study needs to be conducted.

Categories: Endocrinology/Diabetes/Metabolism, Family/General Practice, Other

Keywords: bodyweight, dietary fiber, inulin, guar gum, obese, overweight, weight reduction, bmi, safe, effective

\section{Introduction}

Obesity is a global health concern. Obesity is associated with many diseases, viz., type 2 diabetes mellitus (T2DM), dyslipidemia, atherosclerosis and coronary heart disease, hypertension, and polycystic ovary syndrome (PCOS) $[1,2]$.

It is well known that reduced dietary calorie intake and increased physical activities are useful in reducing bodyweight (BW). Reduced dietary calorie intake is achievable by regulating dietary portion size or reducing calorie-dense food in the diet with the maintenance of the amount of food [3].

There has been an inverse association between dietary fiber consumption and bodyweight [3]. The beneficial effect of the consumption of dietary fiber in obesity appears to be due to its assistance in controlling dietary calorie intake and appetite [4]. In India, there has been diversity in the intake of dietary fiber among different socioeconomic groups. The consumption of dietary fiber appears to be lesser in women [5].

This study was conducted to assess retrospectively the efficacy, safety, and tolerability of dietary fiber supplementation combination (DFSC) in decreasing the bodyweight (BW) and body mass index (BMI) of 


\section{Cureus}

obese or overweight individuals. To our knowledge, this is the first-ever observational study in the world on the assessment of the efficacy and safety of dietary fiber supplementation of a combination of fibers such as inulin, partially hydrolyzed guar gum, and resistant maltodextrin.

\section{Materials And Methods}

\section{Study objective}

To assess the efficacy, safety, and tolerability of dietary fiber supplemental combination (DFSC) of inulin, partially hydrolyzed guar gum, and resistant maltodextrin in obese or overweight cases.

\section{Study design}

This was a retrospective, observational, multicentric, prescription event monitoring study. This study was conducted in accordance with the standard procedures/protocols of conducting retrospective studies [6]. Retrospective data of patients were collected between the period of August 2019 and January 2020. All ethical principles were followed while conducting this study.

\section{Assessment of the test product}

Forty-two overweight to obese individuals consumed dietary fiber supplementation along with dietary and physical activity interventions. The test medication (DFSC) (Fitofy, Nucgnex Lifesciences Private Limited, Pune, India) and its compositions are shown in Table 1.

\begin{tabular}{|c|c|c|c|}
\hline Nutrients & Unit & Per $100 \mathrm{~g}$ & Per serving (6 g) \\
\hline Energy & kcal & 209 & 12.5 \\
\hline Protein & $\mathrm{g}$ & 0 & 0 \\
\hline Fat & g & 0.1 & 0.006 \\
\hline Total carbohydrate & $\mathrm{g}$ & 92 & 5.52 \\
\hline Sugar (sucrose)* & $\mathrm{g}$ & 0 & 0 \\
\hline Soluble dietary tiber & $\mathrm{g}$ & 80 & 4.8 \\
\hline
\end{tabular}

\section{TABLE 1: Composition of dietary fiber supplemental combination}

*No added sugar (natural sugar may come from other ingredients added)

The following dosages of DFSC powder were followed: for obese or overweight patients, 18-24 g DFSC/day (mix one full scoop (6-8 g approximately) of DFSC powder in 250-300 mL water and consume thrice daily (15-20 minutes before meals (pre-lunch and pre-dinner) and 30 minutes pre-bedtime) for 12 consecutive weeks). Approximately $80 \%$ of patients consumed DFSC powder with water with no added sugar, and approximately $20 \%$ of patients consumed it with buttermilk with no added sugar.

\section{Diet pattern}

The following diet patterns before and during the study were followed by most of the patients.

\section{Diet Pattern Before the Study}

Most of the patients were on the following approximate macronutrient pattern of diet before therapy: $60 \%$ $70 \%$ carbohydrate, $8 \%-12 \%$ protein, and $25 \%-30 \%$ fat, with $30 \mathrm{~g}$ visible fat/day and less fiber intake.

Diet Pattern During the Study

Most of the patients were on the following approximate macronutrient pattern of diet during the diet therapy period: $45 \%-55 \%$ carbohydrate, $15 \%-20 \%$ protein, and $20 \%-25 \%$ fat, with $15 \mathrm{~g}$ visible fat/day.

Calories consumed were $1500-1800 \mathrm{kcal} /$ day on weekdays and approximately $2000 \mathrm{kcal} /$ day on weekends for 12 weeks in all patients. All patients were given standard advice regarding healthy food choices in their diet and physical activity. There was vigorous diet monitoring fortnightly for 12 weeks, and a reduction of 200 $\mathrm{kcal} /$ day consumption in the diet was decided on a case-to-case basis if needed by reviewing the calorie consumption by every patient. 
No other nutrient supplementation or weight-reducing medicines were consumed by any patient during 12 weeks of fiber therapy.

\section{Physical activity}

All patients had undergone the following physical activity-related interventions: brisk walking for 30-45 minutes a day for three days in a week and either aerobic exercise, e.g., cardiac exercise/high-intensity exercise (an exercise strategy alternating short periods of anaerobic exercise with less-intense recovery periods until too exhausted to continue, e.g., push-ups, sit-ups, lunges, crunches, and running), or resistant training for 30-45 minutes a day for three days in a week during the study duration. The pattern of physical activity for all patients was similar before and during the study.

\section{Data collection and follow-up}

Observation of Cases

Forty-two overweight to obese individuals were considered for observation in this study at two centers. Data for each subject was documented in a prescription event monitoring form.

Study Visits

The following visits were considered to assess efficacy parameters: first visit, screening day/baseline visit (Day 0); second visit, at the end of four weeks (Day 28); third visit, at the end of eight weeks (Day 56); and fourth visit, at the end of 12 weeks (Day 84). As mentioned before, there was vigorous diet monitoring fortnightly for 12 weeks.

\section{Subject inclusion criteria}

Patients $\geqslant 17$ years of age and both genders were considered. Patients who were overweight or obese were considered for assessment.

\section{Efficacy parameters}

The following parameters were assessed: bodyweight (BW) in $\mathrm{kg}$ and body mass index (BMI). BMI is calculated by dividing an individual's weight in kilograms by the square of height in meters.

\section{Safety assessment}

Patients were evaluated for their safety based on patients' opinions on the tolerability of the DFSC powder and healthcare professionals' opinions on the safety of the DFSC powder.

\section{Concomitant medication}

If any concomitant medication was given to the patient, the same was recorded. Six, one, and two patients were on hypoglycemic, antihypertensive, and anti-hypercholesterolemic medications, respectively.

\section{Statistical analysis}

The statistical analysis of 42 individuals was carried out using the statistical software SPSS version 10.0. The descriptive analysis of demographics and the Student's t-test of significance were used.

\section{Results}

\section{Demographic data}

Demographic data are shown in Table 2. The mean age of the patients was 40.74 years, and the mean bodyweight was $80.63 \mathrm{~kg}$ (Table 2). Female cases were with a higher percentage. 


\section{Cureus}

\begin{tabular}{ll}
\hline Parameters & $\mathbf{N}=\mathbf{4 2}$ \\
Age (years) & \\
Mean \pm SD & $40.74 \pm 12.16$ \\
Range & $18.00-82.00$ \\
Weight $(\mathrm{kg})$ & \\
Mean \pm SD & $80.63 \pm 14.34$ \\
Range & $50.00-117.30$ \\
Gender $(\mathrm{N}(\%))$ & \\
Male & $15(35.7)$ \\
Female & $27(64.3)$
\end{tabular}

\section{TABLE 2: Demographic data}

SD: standard deviation

\section{Efficacy evaluation}

The changes in bodyweight and BMI are shown in Table 3. At the end of weeks 4, 8, and 12, diet therapy and DFSC showed statistically significant reductions in the mean bodyweight and BMI as follows: $3.03 \mathrm{~kg}$ and $1.18 \mathrm{~kg} / \mathrm{m}^{2}, 5.70 \mathrm{~kg}$ and $2.31 \mathrm{~kg} / \mathrm{m}^{2}$, and $7.82 \mathrm{~kg}$ and $3.27 \mathrm{~kg} / \mathrm{m}^{2}$, respectively. The results are depicted in Figure 1 and Figure 2.

\begin{tabular}{|ll} 
Duration in weeks & Mean bodyweight $(\mathbf{k g})$ \\
\hline Baseline & $80.63 \pm 14.34$ \\
4 & $78.51 \pm 13.67$ \\
8 & $75.61 \pm 13.57$ \\
12 & $72.81 \pm 13.25$ \\
Mean diff (baseline - week 4$)(p$ value) & $-03.03 \pm 01.24(0.001)^{*}$ \\
Mean diff (baseline - week 8$)(p$ value) & $-05.70 \pm 02.21(0.001)^{*}$ \\
Mean diff (baseline - week 12$)(p$ value) & $-07.82 \pm 03.06(0.001)^{*}$
\end{tabular}

Mean BMI $\left(\mathrm{kg} / \mathrm{m}^{2}\right)($ mean $\pm \mathrm{SD})(\mathrm{N}=42)$

$32.24 \pm 13.98$

$31.53 \pm 14.24$

$30.32 \pm 13.60$

$28.97 \pm 12.57$

$-01.18 \pm 00.52(0.001)^{*}$

$-02.31 \pm 01.08(0.001)^{*}$

$-03.27 \pm 01.86(0.001)^{*}$

TABLE 3: Changes in the bodyweight and body mass (BMI) with diet therapy and DFSC

Mean diff: mean difference (Student's t-test); *: significant difference; SD: standard deviation; DFSC: dietary fiber supplemental combination 


\section{Cureus}

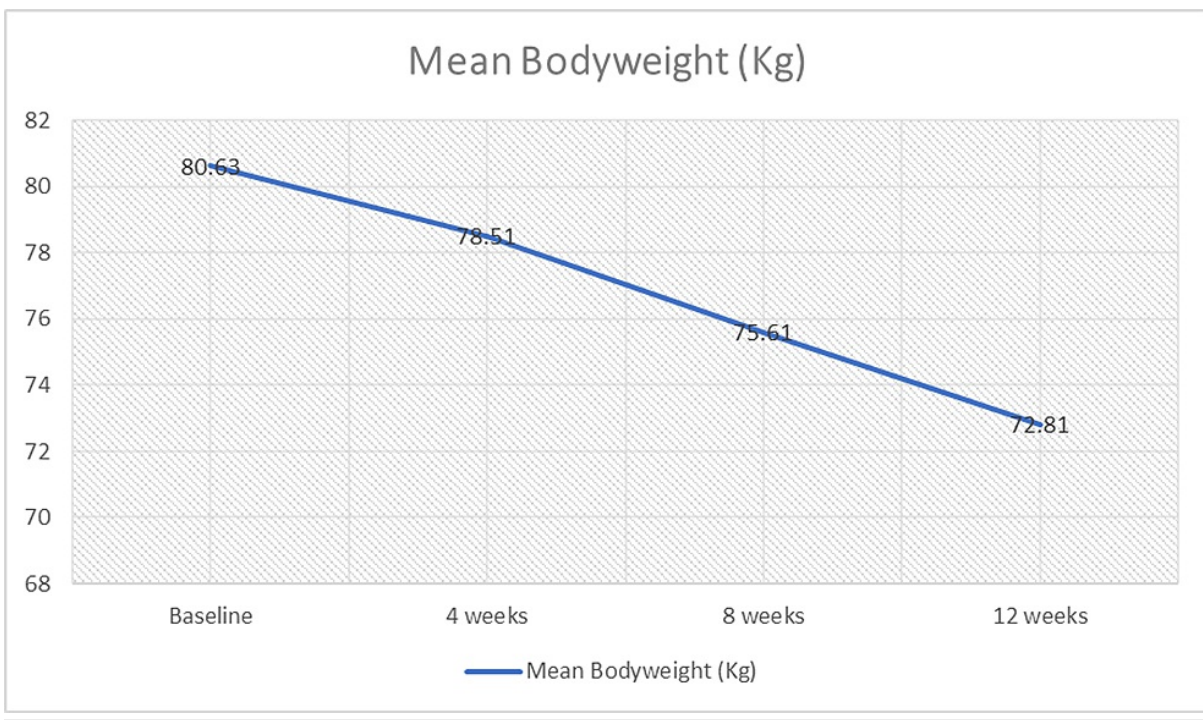

FIGURE 1: Changes in the mean bodyweight among the study cases with diet therapy and DFSC

DFSC: dietary fiber supplemental combination

\section{MEAN BMI $\left(\mathrm{kg} / \mathrm{m}^{2}\right)$}

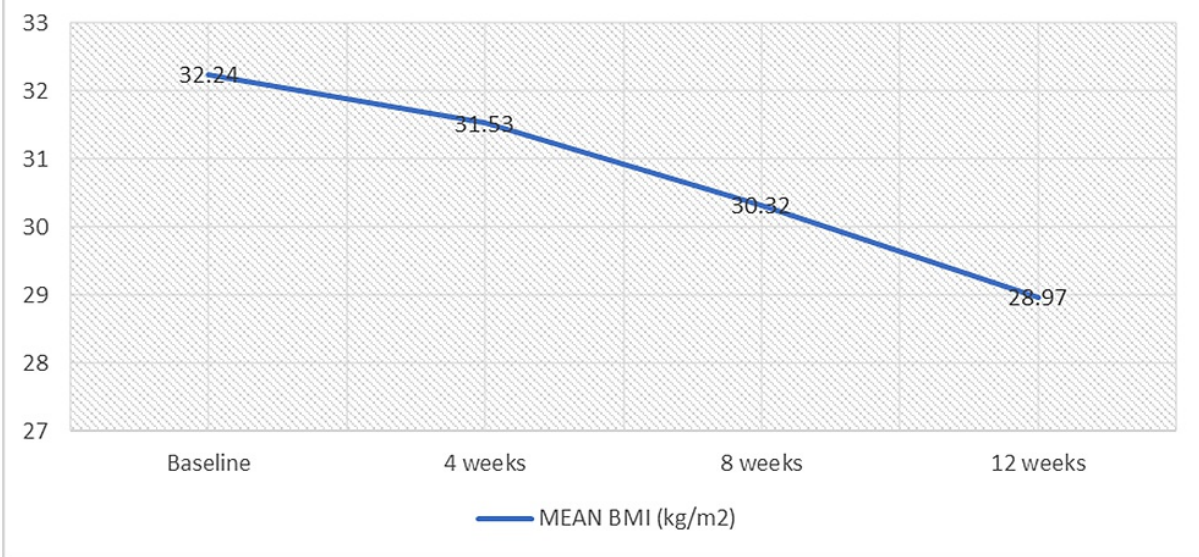

FIGURE 2: Changes in the mean body mass (BMI) among the study cases with diet therapy and DFSC

DFSC: dietary fiber supplemental combination

Evaluation of tolerability, safety, and efficacy of DFSC by patients and healthcare professionals

Patients' opinions on tolerability and efficacy are shown in Table 4. A view of good to excellent tolerability with DFSC was opined by $95.3 \%$ of the patients, and $100 \%$ of the patients opined that diet therapy along DFSC has good to excellent efficacy.

Healthcare professionals' opinions on safety and efficacy are demonstrated in Table 5. As per the healthcare professionals, good to excellent safety with DFSC was observed in $97.6 \%$ of the cases, adverse event was not reported in any patient, and good to excellent efficacy with diet therapy and DFSC was observed in $97.6 \%$ of the patients. 


\section{Cureus}

Patients' opinions on DFSC tolerability

\begin{tabular}{|c|c|c|}
\hline Opinion & No. of cases $(\mathrm{N}=42)$ & Percentage \\
\hline Poor & - & - \\
\hline Fair & 02 & 4.7 \\
\hline Good & 26 & 62 \\
\hline Excellent & 14 & 33.3 \\
\hline \multicolumn{3}{|c|}{ Patients' opinions on diet therapy and DFSC efficacy } \\
\hline Opinion & No. of cases $(\mathrm{N}=42)$ & Percentage \\
\hline Poor & - & - \\
\hline Fair & - & - \\
\hline Good & 28 & 66.6 \\
\hline Excellent & 14 & 33.4 \\
\hline
\end{tabular}

\section{TABLE 4: Patients' opinions on tolerability and efficacy}

DFSC: dietary fiber supplemental combination

Healthcare professionals' opinions on DFSC safety

\begin{tabular}{|c|c|c|}
\hline Opinion & No. of cases $(\mathrm{N}=42)$ & Percentage \\
\hline Poor & - & - \\
\hline Fair & 01 & 2.4 \\
\hline Good & 07 & 16.6 \\
\hline Excellent & 34 & 81 \\
\hline \multicolumn{3}{|c|}{ Healthcare professionals' opinions on diet therapy and DFSC efficacy } \\
\hline Opinion & No. of cases $(\mathrm{N}=42)$ & Percentage \\
\hline Poor & - & - \\
\hline Fair & 01 & 2.4 \\
\hline Good & 34 & 81 \\
\hline Excellent & 7 & 16.6 \\
\hline
\end{tabular}

TABLE 5: Healthcare professionals' opinions on safety and efficacy

DFSC: dietary fiber supplemental combination

\section{Discussion}

It is concluded in "The Finnish Diabetes Prevention Study" that long-term weight loss can be achieved with a high-fiber and low-fat diet as it was found to be greater in reducing bodyweight than that with a high-fat and low-fiber diet with results of approximately $3 \mathrm{~kg}$ versus $0.7 \mathrm{~kg}$ bodyweight reduction, respectively, in three years. Researchers also claimed that a low-fat, low-energy-density and high-fiber diet caused continued bodyweight reduction. The same study reported that the low-carbohydrate diet was more effective than the conventional low-fat and low-energy diet in causing significant short-term weight reduction. This might be due to the overall energy deficiency [3]. The weight-reducing effect observed in the current study is consistent with "The Finnish Diabetes Prevention Study"; although the patients in the present study consumed a low-fiber diet, it was compensated with the consumption of DFSC, and that may 
In a six-month study conducted by Cicero et al. in hypertensive and overweight patients, oral consumption of guar gum 20 minutes before meals (nearly $10 \mathrm{~g}$ /day divided into three doses) resulted in significant improvement in the BMI, fasting plasma glucose (FPG) and insulin (FPI) levels, glycosylated hemoglobin (HbA1c), homeostasis model assessment (HOMA) index, low-density lipoprotein cholesterol (LDL-C), and apolipoprotein B (ApoB) [7]. The BMI-reducing effect was also observed in the present study with diet therapy and guar gum containing DFSC.

As per the review by Howarth et al., various studies demonstrate the following: increased consumption of soluble fiber causes increased after-meal satiety effect and reduces consequent hunger, increased fiber intake leads to a weight reduction of approximately $2 \mathrm{~kg}$ in about four months, and a reduction in energy intake and bodyweight takes place with natural high-fiber diet or with a dietary fiber supplement [7]. Various studies conducted by Howarth et al., Liu et al., and Ludwig et al. indicate that a higher intake of dietary fiber is associated with bodyweight reduction [8-10]. As per a review by Anderson et al., the recommended dietary fiber intakes for children and adults are $14 \mathrm{~g} / 1000 \mathrm{kcal}$ [11].

In our study, diet therapy and DFSC (containing soluble fiber) showed significant reductions in the mean bodyweight. The patients were not on any bodyweight-reducing medicines, and no patient needed such medicines during the consecutive 12 weeks of fiber therapy. Excellent tolerability and safety profile with DFSC were observed. These results with DFSC are very encouraging. However, a randomized controlled study is warranted to evaluate the efficacy of DFSC. The physical activity may not have contributed much to significant reductions in the mean bodyweight and BMI as the pattern of physical activity for all the patients was similar before and during the study. Calorie restriction was not strictly followed by all the patients before getting involved in the study, and calorie-restricted diet consumption was strictly monitored for all the patients during the study, which appeared to have contributed to significant reductions in the mean bodyweight. The weight-reducing effect appears to be superior to that observed in previously conducted fiber-related studies. A superior effect may be due to fortnightly vigorous diet monitoring for 12 weeks, including a reduction of $200 \mathrm{kcal} /$ day consumption in the diet on a case-to-case basis.

The following could be the mechanisms behind the beneficial use of dietary fiber for reducing bodyweight. Preclinical studies have shown that the ingestion of inulin-type fructans can regulate bodyweight via the promotion of endogenous glucagon-like peptide-1 (GLP-1) in the gut. GLP-1 is the key controller of food intake by promoting satiety that consequently reduces the intake of food to cause a decrease in bodyweight and BMI [12]. As per the review by Howarth et al., a direct effect of dietary fiber on the gastric distension or delayed gastric emptying leads to the sensation of fullness; dietary fiber has an indirect effect by secreting intestinal hormones, e.g., incretins; fibers may cause reduced energy intake by reducing the absorption of fat and protein [8]. A study by Lyly et al. concluded that guar gum fiber-containing beverage showed a greater satiety effect and lower desire to eat than that with the beverage without fiber [4]. In a randomized controlled trial, Pol et al. reported a reduction in the appetite with the consumption of prebiotic dietary fiber (obtained from chicory root inulin) containing oligofructose granola bars as snack replacements in overweight and obese adults [13].

In the present study, the satiety effect of dietary fiber supplemental combination was not evaluated. Although few patients from the current study communicated that their appetite was reduced, it needs to be evaluated further.

The following additional benefits were found with dietary fiber in various studies. Liu et al. concluded that inulin appears to reduce LDL-C among patients suffering from overweight or obesity, metabolic abnormalities including dyslipidemia, and T2DM, whereas glucose control and HDL-C improvement seem to be reported in T2DM cases [14]. As per a study conducted by Dall'Alba et al., the supplementary partially hydrolyzed guar gum (PHGG) improved metabolic and cardiovascular profiles due to reduction in glycated $\mathrm{Hb}$ (HbA1c), waist circumference (WC), serum trans-fatty acids (FA), and 24-hour urinary albumin excretion (UAE) [15]. As per Blake et al., depolymerized guar gum-supplemented wheat bread reduces plasma cholesterol concentration in patients with hypercholesterolemia [16]. They also concluded that resistant maltodextrin seems to improve the risk factors of metabolic syndrome by improving glucose and lipid metabolism and by reducing visceral fat [16]. However, most of the parameters from the abovementioned studies, excluding bodyweight and BMI, were not assessed in the present study.

The current study is the first-ever study on the assessment of the effect of DFSC of inulin, partially hydrolyzed guar gum, and resistant maltodextrin. We hypothesize that the contents of DFSC may be a synergistic combination of fibers to support bodyweight-reducing effect with diet therapy, which needs to be evaluated further.

\section{Limitations of the study}

The efficacy of DFSC was assessed for three months only, and a long-term assessment of the efficacy is needed. This was an observational, uncontrolled, retrospective, prescription event monitoring study. Other 
overweight- or obesity-associated parameters, viz., body fat area, waist circumference, satiety effect, blood glucose parameters, and lipid profile, were not assessed during the study. Diet and physical activity are possible confounding factors in evaluating the effect of individual dietary components/supplements such as fiber. A randomized, controlled clinical trial in a greater number of subjects with control of all the confounding factors is warranted to evaluate the efficacy of the dietary fiber supplementation combination and to confirm the synergistic effect of this combination.

\section{Conclusions}

As per this observational, retrospective, prescription event monitoring study, the dietary fiber supplemental combination of inulin, partially hydrolyzed guar gum, and resistant maltodextrin (DFSC) with proper diet therapy/modification is found to be safe and effective in causing significant weight reduction in obese or overweight patients. However, a large multicentric, randomized controlled study in a wider range of patients and a larger number of patients including more investigational parameters for longer duration needs to be conducted with ruling out possible confound factors.

\section{Additional Information \\ Disclosures}

Human subjects: Consent was obtained or waived by all participants in this study. Animal subjects: All authors have confirmed that this study did not involve animal subjects or tissue. Conflicts of interest: In compliance with the ICMJE uniform disclosure form, all authors declare the following: Payment/services info: All authors have declared that no financial support was received from any organization for the submitted work. Financial relationships: All authors have declared that they have no financial relationships at present or within the previous three years with any organizations that might have an interest in the submitted work. Other relationships: All authors have declared that there are no other relationships or activities that could appear to have influenced the submitted work.

\section{References}

1. Boschmann M, Thielecke F: The effects of epigallocatechin-3-gallate on thermogenesis and fat oxidation in obese men: a pilot study. J Am Coll Nutr. 2007, 26:389S-95S. 10.1080/07315724.2007.10719627

2. Sam S: Obesity and polycystic ovary syndrome. Obes Manag. 2007, 3:69-73. 10.1089/obe.2007.0019

3. Lindström J, Peltonen M, Eriksson JG, Louheranta A, Fogelholm M, Uusitupa M, Tuomilehto J: High-fibre, low-fat diet predicts long-term weight loss and decreased type 2 diabetes risk: the Finnish Diabetes Prevention Study. Diabetologia. 2006, 49:912-20. 10.1007/s00125-006-0198-3

4. Lyly M, Liukkonen KH, Salmenkallio-Marttila M, Karhunen L, Poutanen K, Lähteenmäki L: Fibre in beverages can enhance perceived satiety. Eur J Nutr. 2009, 48:251-8. 10.1007/s00394-009-0009-y

5. Position of the Indian Dietetic Association: dietary fibre and health . (2018). http://idaindia.com/wpcontent/uploads/2018/12/IDA-position-paper-fibre-24.12.18.pdf.

6. Motheral B, Brooks J, Clark MA, et al.: A checklist for retrospective database studies--report of the ISPOR Task Force on Retrospective Databases. Value Health. 2003, 6:90-7. 10.1046/j.1524-4733.2003.00242.x

7. Cicero AF, Derosa G, Manca M, Bove M, Borghi C, Gaddi AV: Different effect of psyllium and guar dietary supplementation on blood pressure control in hypertensive overweight patients: a six-month, randomized clinical trial. Clin Exp Hypertens. 2007, 29:383-94. 10.1080/10641960701578378

8. Howarth NC, Saltzman E, Roberts SB: Dietary fiber and weight regulation. Nutr Rev. 2001, 59:129-39. 10.1111/j.1753-4887.2001.tb07001.x

9. Liu S, Willett WC, Manson JE, Hu FB, Rosner B, Colditz G: Relation between changes in intakes of dietary fiber and grain products and changes in weight and development of obesity among middle-aged women. Am J Clin Nutr. 2003, 78:920-7. 10.1093/ajcn/78.5.920

10. Ludwig DS, Pereira MA, Kroenke CH, Hilner JE, Van Horn L, Slattery ML, Jacobs DR Jr: Dietary fiber, weight gain, and cardiovascular disease risk factors in young adults. JAMA. 1999, 282:1539-46. 10.1001/jama.282.16.1539

11. Anderson JW, Baird P, Davis RH Jr, et al.: Health benefits of dietary fiber. Nutr Rev. 2009, 67:188-205. 10.1111/j.1753-4887.2009.00189.x

12. Yeo SK, Ooi LG, Lim TJ, Liong MT: Antihypertensive properties of plant-based prebiotics. Int J Mol Sci. 2009, 10:3517-30. 10.3390/ijms10083517

13. Pol K, de Graaf C, Meyer D, Mars M: The efficacy of daily snack replacement with oligofructose-enriched granola bars in overweight and obese adults: a 12-week randomised controlled trial. Br J Nutr. 2018, 119:1076-86. 10.1017/S0007114518000211

14. Liu F, Prabhakar M, Ju J, Long H, Zhou HW: Effect of inulin-type fructans on blood lipid profile and glucose level: a systematic review and meta-analysis of randomized controlled trials. Eur J Clin Nutr. 2017, 71:9-20. 10.1038/ejcn.2016.156

15. Dall'Alba V, Silva FM, Antonio JP, et al.: Improvement of the metabolic syndrome profile by soluble fibre guar gum - in patients with type 2 diabetes: a randomised clinical trial. Br J Nutr. 2013, 110:1601-10. $10.1017 /$ S0007114513001025

16. Blake DE, Hamblett CJ, Frost PG, Judd PA, Ellis PR: Wheat bread supplemented with depolymerized guar gum reduces the plasma cholesterol concentration in hypercholesterolemic human subjects. Am J Clin Nutr. 1997, 65:107-13. 10.1093/ajcn/65.1.107 